\title{
LESS INVASIVE STABILIZATION SYSTEM (LISS) IN THE TREATMENT OF DISTAL FEMUR FRACTURES
}

\author{
N. Dimitrov ${ }^{*}$, D. Petrov², G. Mratskova ${ }^{3}$, I. Dimitrov ${ }^{4}$ \\ ${ }^{1}$ Department of Orthopedics and Traumatology, University Hospital "Prof. Dr. St. Kirkovich", \\ Stara Zagora, Bulgaria \\ ${ }^{2}$ Department of Social Medicine and Health Management, Medical Faculty, Trakia University, \\ Stara Zagora, Bulgaria \\ ${ }^{3}$ Department of Medical Rehabilitation and Ergotherapy, Physical medicine and Sport, \\ Medical Faculty, Trakia University, Stara Zagora, Bulgaria \\ ${ }^{4}$ Medizinische Fakultat, Friedrich - Schiller - Universitst, Jena, Germany
}

\begin{abstract}
Purpose. To rewiew the advantages of LISS for complex distal femoral fractures.

Methods. 25 women and 19 men underwent surgical LISS plating for distal femur fractures following low- or high-energy injury between $2011-09.2015$. The mean age of patients was $53.34 \pm 20.1$ years. One patient had a pathologic femur fracture because of metastasis. One patient had a bilateral femoral fracture following a traffic accident. In one patient a periprosthetic femoral fracture occurred after total knee arthroplasty. According to the AO classification, predominantly occurring fracture types were A1 $(38.1 \%)$ and C1 $(35.7 \%)$.

Results. The mean period of bone union was $3.4 \pm 1$ months. Two patients (4.8\%) had non-union due to implant loosening and required revision surgery and osteosynthesis RFN. One case (2.4\%) with deep infection necessitating revision surgery with debridement, jet-lavage, drainage, combined antibiotic therapy and application of gentamycin beads. One patient $(2.4 \%)$ with delayed bone healing. One patient $(2.4 \%)$ underwent primary bone grafting in AO C2 fracture.

Conclusion. All of the distal femur fractures A - C of the AO, incl. and all degrees of severity of these fractures can be successfully stabilized by LISS.
\end{abstract}

Key words: femoral fractures, internal fracture fixation, mini invasive techniques

\section{INTRODUCTION}

A noticeable evolution of distal femur treatment concepts has occurred over the past decades as innovative implants and improved minimal invasive indirect reposition techniques developed. The main priorities shifted towards rapid fracture union, angular alignment and precise joint congruency, early active joint mobilization and patient verticalization. The advantages of such developments that focus on these goals are increasingly popular in recent years.

The LISS (Less Invasive Stabilization System) is modern method of internal fixation which incorporates the biomechanical advantages of lateral stabilization of distal femur fractures

\footnotetext{
*Correspondence to: $N$. Dimitrov Department of Orthopedics and Traumatology, University Hospital "Prof. Dr. St. Kirkovich”, Stara Zagora, Bulgaria,E-mail: dimitrovnedko@yahoo.com
}

with the principles of optimal bone-to-implant contact $(1,2)$. The state of the art concept utilizes a classical ("open") reduction of fragments under direct visualisation of the joint components, combined with a "closed" manipulation of metaphyseal/diaphyseal by reduction aiding instruments. This allows the system to act more as an "internal fixator" than a conventional plate $(3,4)$.

\section{MATERIALS AND METHODS}

A consecutive series of 44 patients with a mean age of $53.34 \pm 20.1$ (mean \pm SD), (range $18-91$ ) years were evaluated. 25 women $(56.8 \%)$ and 19 men (43.2\%), (matched 1.3:1) were all hospitalized for emergency treatment in the department of Orthopedy and Traumatology of the University hospital "Prof. Stoyan Kirkovitch" $\mathrm{AD}$, Stara Zagora and underwent surgical LISS plating for distal femur fractures following lowor high-energy injury between 2011 and 09.2015. Table 1 summarizes the patient demographics in the series. 
Table 1. Demographic characteristics of patients in this study.

\begin{tabular}{|l|l|}
\hline Demographic characteristics & Values \\
\hline Age (years) mean \pm SD (range) & $53.34 \pm 20.1(18-91)$ \\
Men & $45.84 \pm 16.7(18-73)$ \\
Women & $63.21 \pm 20.3(22-91)$ \\
\hline Gender & $\mathrm{N}=19(43.2 \%)$ \\
Men & $\mathrm{N}=25(56.8 \%)$ \\
Women & \\
\hline Hospital stay (days) mean \pm SD (range) & $8.7 \pm 3(6-18)$ \\
Men & $9.0 \pm 3.1(6-17)$ \\
Women & $8.4 \pm 3.1(6-18)$ \\
\hline
\end{tabular}

Patients who were above 50 years of age made up $79 \pm 6.14 \%$ of the women group; whereas $56 \pm 7.5 \%$ of the men were aged less than 50 . One patient had a pathologic femur fracture because of metastatic bone disease in the distal femur. One patient had a bilateral femur fracture following a traffic accident. In one patient a periprosthetic femur fracture occurred after total knee arthroplasty.

LISS implants and corresponding instruments from "Synthes" and "Mahe Medical GmbH Germany" were used for the operative treatment of the fractures. 20 patients' 7-hole distal femur plates, 9-hole, 11-hole. Depending on the fracture pattern, 5-, 9-, or 13-hole LISS plates were applied. Used plates were: seven in 20 patients $((47.62 \pm 7.71) \%)$, nine in 17 patients((40.48 \pm 7.57$) \%)$ and eleven in 5 patients $((11.9 \pm 5.0) \%)$ hole: total - 42 patients +2 patient with screw osteosynthesis (AO type B - fractures). Patients with B-type (monocondyle) fractures were excluded from this series, as these were treated with conventional screw osteosynthesis.

Surgical treatment included open reduction and internal fixation of interarticular fragments, indirect reduction of metaphyseal and metadiaphyseal fractures with MIPPO (minimally invasive percutaneous plate osteosynthesis). An open reduction technique was carried out in 10 patients. LISS-plate was fixed between muscle and metaphysis/diaphysis in a submuscular tunnel using minimally invasive techniques under $\mathrm{x}$ ray imaging with less bone exposure and leaving soft tissue surrounding the fracture site intact. The following surgical approaches were used: lateral MIPPO $50 \pm 7.72 \%$, paralateral MIPPO $26 \pm 6.78 \%$ and open technique $24 \pm 6.59 \%$. The operative intervention took place in up to three days after initial hospitalization. 23 of all cases during the first 24 hours (58.8 \pm 7.6$)$ and 19 after 24 hours $(45.2 \pm 7.6 \%)$. Patients who did not undergo surgery in the first 24 hours were put on direct skeletal extension through tuberositas tibiae. Patients with polytrauma and combined fractures were operated after shock management and vital function stabilization .

Predominantly occurring fracture types in this study were AO types A1 and $\mathrm{C} 1$ with $38.1 \pm 7.5 \%$ and $35.7 \pm 7.4 \%$ respectively. Fractures of the types A2, A3 and C2 accounted for $7.1 \pm 4.0 \%$ each from all patients. C3-type fractures had the lowest incidence $4.8 \pm 3.3 \%$. Furthermore distal femur fractures were grouped by mechanism of injury in lowand high-energy fractures. Those with a highenergy mechanism of injury were more common. They made up $2 / 3$ of all fractures in the series and were mostly due to traffic accidents and falls. The low-energy mechanism of injury group comprised of $85 \%$ women and $15 \%$ men. In comparison highenergy trauma included mostly men.

In our patients injuries were mostly closed and only $7 \%$ - were open fractures, with superficial wound Gustillo grade I - II. In 30\% of cases the fracture of the distal femur was combined with other musculoskeletal injuries: patellar fracture $(n=2)$, fracture of the proximal tibia, ligament injury in the knee, pelvis fracture, fracture of the proximal humerus and clavicula, and distal antebrachium fractures. Open fractures underwent urgent surgical treatment (incl. irrigation, debridement, fixation, antibiotic medication).

The mean duration of surgery was $92.5 \mathrm{~min}$. (50 to $135 \mathrm{~min}$.) in type-A fractures and 130 min. (60 to $200 \mathrm{~min}$ ) in type-C fractures.

$\mathrm{X}$-ray imaging exposure times in the course of surgery were: 3.0 to $17.0 \mathrm{~min}$ for A-type fractures (22 patients); 2.5 to $15.0 \mathrm{~min}$ for $\mathrm{C}$ type fractures (20 patients).

Postoperative patient mobilization took place on the next day following surgery and was performed by a kinetic physical therapist with 
DIMITROV N., et al.

no weight bearing and walking two aids. Postoperative knee mobilization began on the second and third days after surgery. Polytrauma patients on assisted breathing (intubated) in the intensive care unit (ICU) were mobilized by means of passive motion machine (CPM).

All patients in this study were followed up at regular intervals until satisfactory union for a period of 2 to 24 months. Monthly radiographic (x-ray) and clinical control was performed. Operative treatment was considered successful when radiological and clinical evidence of union until the $12^{\text {th }}$ month was assessed.

\section{RESULTS}

The mean time to full weight bearing was dependent of the type of fracture. A1-type fractures allowed for partial weight bearing after an initial postoperative period of 7 to 10 days. Partial weight bearing by comminuted and multiple fractures of AO types A3 and C began between the $30^{\text {th }}$ to $45^{\text {th }}$ postoperative days. Stability in the latter is provided primarily by the LISS plate. Premature full weight bearing poses the risk to overstress the construct and compromise the mechanical stability of the implant.

Full weight bearing began depending on the clinical and radiological progress of fracture healing and subjective patient comfort. Patients were followed up at regular intervals for functional assessment until full union. Fractures were considered healed when evidence of trabecular cortical bridging was documented. Practically no periosteal callus was observed aside from the cases with malalignment and instable fixation.

The mean period of bone union in the evaluated group was $3.4 \pm 1$ months (2-5 months) - Table 2:

Table 2. The mean period of bone union

\begin{tabular}{|l|l|l|}
\hline Time to union, (months) & Num. of cases & Percentage \pm Sp \\
\hline$\leq 3$ months & 24 & $57.1 \pm 7.6 \%$ \\
\hline $4-5$ months & 15 & $35.7 \pm 7.4 \%$ \\
\hline Nonunion & 2 & $4.8 \pm 3.3 \%$ \\
\hline Delayed union & 1 & $2.4 \pm 2.4 \%$ \\
\hline
\end{tabular}

Complication we encountered requiring revision surgery in this series included:
1. Two patients (4.8\%) had non-union due to implant loosening and required revision surgery and osteosynthesis RFN (Figure 1)

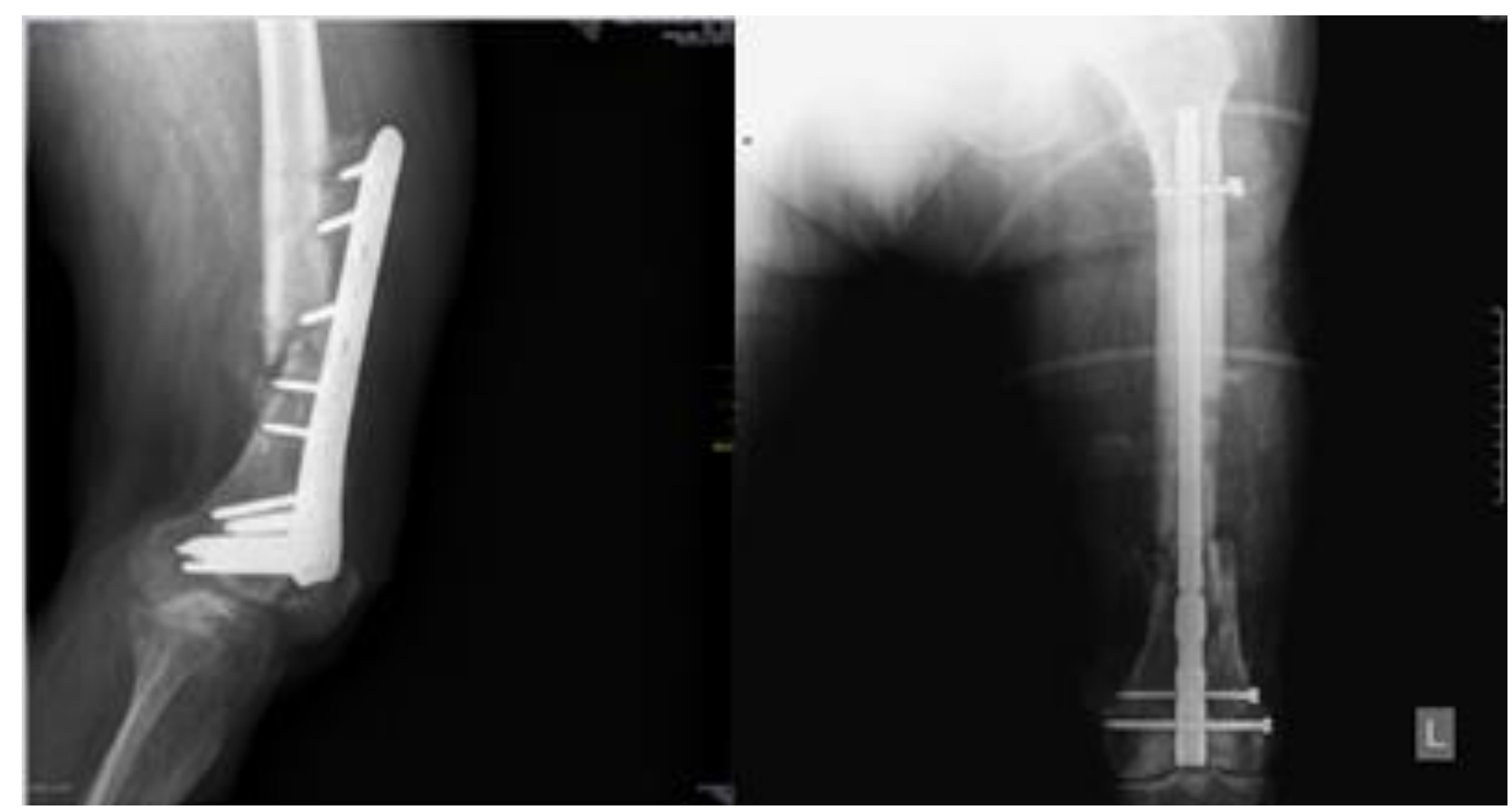

Figure 1. Implant loosening 
2. One case $(2.4 \%)$ with deep infection necessitating revision surgery with debridement, jet-lavage, drainage, double
DIMITROV N., et al. combined antibiotic therapy and application of gentamycin beads (Figure 2).

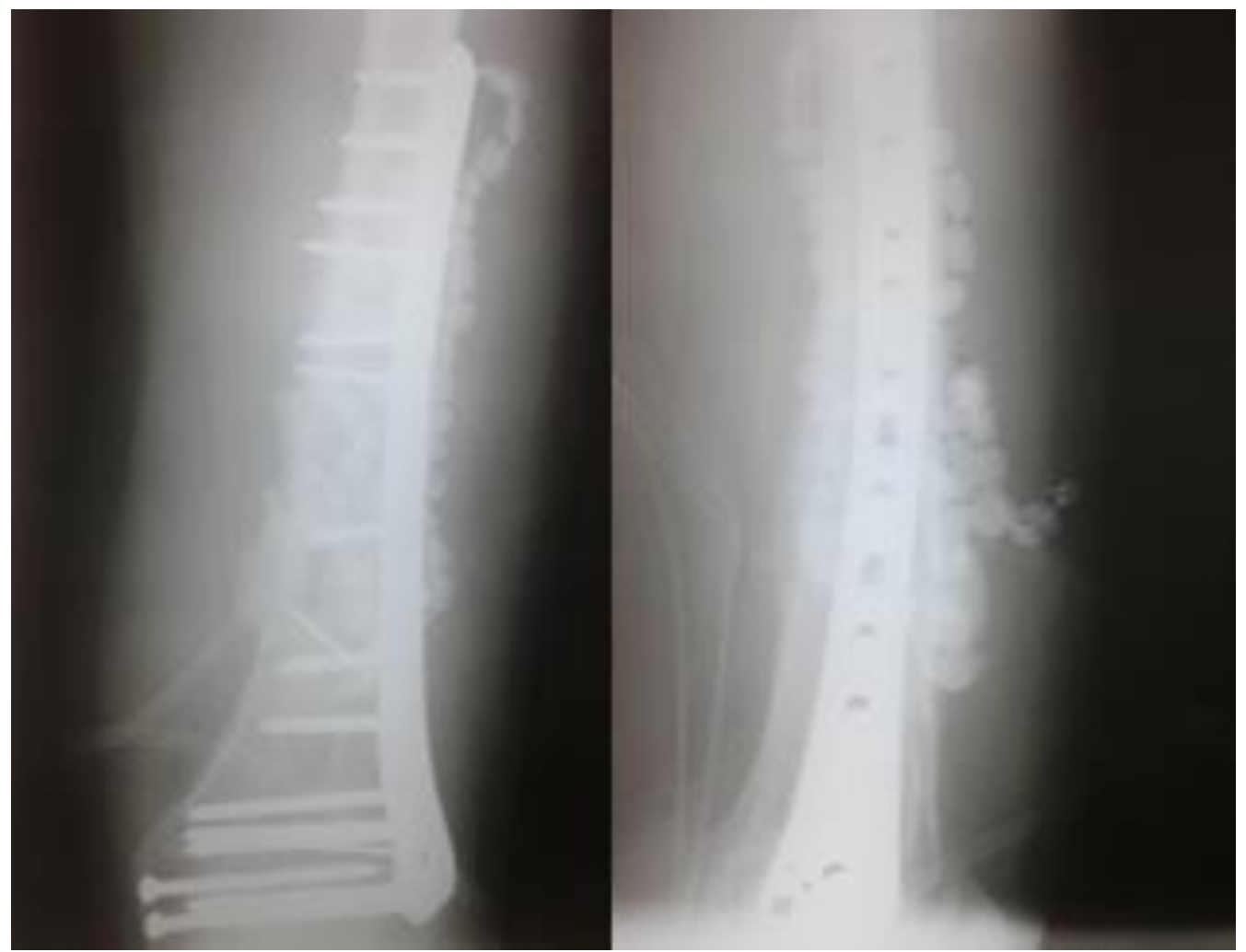

Figure 2. Local application of gentamycin beads

3. One patient (2.4\%) with delayed bone healing

4. One patient (2.4\%) underwent primary bone grafting in an $\mathrm{AO} \mathrm{C} 2$ fracture.

5. Without neurological and vascular complications in the follow-up

Implant loosening was only observed in the proximal attachment of the plate to the femoral dyaphysis. The reasons are improper fit of the implant on the femoral diaphysis (in ventral) and too greater offset LISS-plate of the femoral diaphysis with incorrectly located cortical screws, improper locking of the screws to the implant, thereby losing the effect of "internal fixator." Another reason is the failure to comply with weight bearing restrictions.

In $39(92.9 \pm 4.0) \%$ cases evidence of correct reduction was documented on both anteroposterior and lateral radiographs. In 3 cases $(7.1 \pm 4.0) \%$ dorsal $(n=1)$ and ventral $(n=2)$ drift of the implant to the femoral diaphysis was observed. In 2 cases $(4.8 \pm 3.3) \%$ we had the incorrect location of the implant to the femoral condyles. Postoperative evaluation of fracture regarding varus / valgus was in a good position at $37(88.1 \pm 5.0) \%$ cases. In three $(7.1 \pm 4.0) \%$ cases there was variation in the anatomy of 5 to $10^{\circ}$ varus / valgus and one $(2.4 \pm 2.4 \%)$ case there was a deviation in the 10 to $20^{\circ}$. In the sagittal plane there were two $(4.8 \pm 3.3) \%$ cases with a tolerance of 5 to $10^{\circ}$ and one $(2.4 \%)$ case - from 10 to $20^{\circ}$. Two $(4.8 \% \pm 3.3) \%$ patients had an external rotary deflection relative to the contralateral side.

By the end of follow-up, 40 patients $((95.2 \pm 3.3) \%)$ had completely united fractures, with the ability of full weight bearing of the limb. In two patients $(4.8 \pm 3.3) \%$ ) no union was achieved until the end of this study.

Table 3. Distribution of patients according to range of knee flexion after LISS-plating

\begin{tabular}{|l|l|l|}
\hline Degrees of flexion & Count & Percentage \pm Sp \\
\hline$<95$ & 9 & $21.4 \pm 6.3$ \\
\hline $95-110$ & 11 & $26.2 \pm 6.8$ \\
\hline$>110$ & 22 & $52.4 \pm 7.7$ \\
\hline Total & 42 & 100.0 \\
\hline
\end{tabular}


Satisfactory flexion, compatible with everyday activity is $110^{\circ}$; mean flexion in our series was $106^{\circ} \pm 11.6^{\circ}$.

The results were evaluated according to the Neer's criteria and scores for supracondylar femur fractures:

In $50 \%$ of all patients assessed knee flexion was greater than $110^{\circ}$. Neer scores we achieved in this series of 42 patients were as follows:

- $65 \%$ - excellent result

- $20 \%$ - good result

- $10 \%$ - fair result

- $5 \%$ - poor result

\section{DISCUSSION}

Distal femoral fractures normally exhibit a bimodal mechanism of occurrence: (5) as a result of high-energy trauma in younger patients, associated with higher incidence of comminuted and open and (6) low-energy mechanism in older patients with osteopenic or osteoporotic bones. In our study group has been retained this bimodal distribution pattern of fractures of the distal femur - low-energy fractures, mainly in women over '50 age, and high-energy fractures - in young men.

Mean range of knee flexion postoperative achieved for the entire series was $106^{\circ} \pm 11.6^{\circ}$, accounting to $78.5 \%$ of normal flexion in normal individuals. Results we obtained of varus/ valgus, flexion and extension in the knee joint are comparative to other LISSplating studies (7).

Mean time of union achieved was $3.4 \pm 1$ months (2 -5 months) in all patients in this study. In correlation with the type of reductionMIPPO or open surgical technique, times until full union differ: MIPPO - techniques help faster bone union $-\mathrm{Me}=3(2-5)$ months than the open technique to reposition $-\mathrm{Me}=4(3.5$ to 5) months. Bone biology is considered to be preserved as no bone fragments were reported to be de-periotstated and blood supply was preserved.

Similar results were reported in literature with a $100 \%$ of postoperative bone healing in acute fractures for a mean of 3.5 months(8-10).

We did not observe any loss of fixation in 12 of a total of 13 elderly patients, despite advanced stage osteoporosis and age higher than 65 .

Low rate of infectious complications was reported throughout the whole series (only one case of deep infection; no cases of superficial infection), despite the presence of 3 open fractures (Gustilo I - II grade). This is attributed to the standard preoperative antibiotic prophylaxis and the early debridement performed at the fracture site but also to the minimally invasive surgical technique of LISS-plate positioning. Other studies reported comparable results (1). Deep infections required secondary operative treatment - lavage, drainage and antibiotic treatment according to our regimen.

Traditional bone grafting used to be accepted as a way to replace non-vital bone fragments caused by open techniques of fracture treatment. This is rarely necessitated when minimally invasive techniques are applied that protects the soft tissue anatomy of the fracture site. In our study a bone graft from crista illiaca was required in one patient with $\mathrm{C} 2$ type fracture, suffering from severe osteoporosis with subchondral bone loss due to spongiosa impacting. No further complications were observed in this patient and a satisfactory final outcome was achieved.

LISS - the system is convenient and easy to work with proven practical advantages such as minimal invasive operating techniques. Short times to bone union with the addition of minimal soft tissue disruption and preservation of blood supply to the bone makes LISSplating preferable for comminuted intraarticular fractures and a priority for osteoporotic, periprosthetic and other related fractures. Due to the closed (submuscular) technique a low degree of postoperative infectious complications was reported. It provides angular and axial stability, without the need to use autogenous bone graft in 95\% of cases, and a stable distal femoral support $100 \%$, even in osteoporotic bones.

The treatment of complex distal femur fractures is demanding and requires a thorough understanding of fracture biology. All of the distal femur fractures $\mathrm{A}-\mathrm{C}$ of the AO, incl. and all degrees of severity of these fractures can be successfully stabilized by LISS.

\section{REFFERENCES}

1. Krettek, C.; Schandelmaier, P.; Miclau, T. et al. Transarticular joint reconstruction and indirect plate osteosynthesis for complex distal supracondylar femoral fractures. Injury, 1:A31-41, 1997.

2. Krettek, C.; Schandelmaier, P.; Miclau, T. and Tscherne, H. Minimally invasive percutaneous plate osteosynthesis (MIPPO) using the CS in proximal and distal femoral fractures. Injury, 1:A20-30, 1997. 
3. Mast, J.; Jakob, R. and Ganz, R. Planning and reduction techniques in fracture surgery. New York: Springer-Verlag, 1989.

4. Muller, M.E. et al. The comprehensive classification of fractures of long bones. In Muller, M.E.; Allgower, M.; Schneider, R.,; Willenneger, H. (eds). Mannual of internal fixation. $3^{\text {rd }}$ ed Berlin Springer-Verlag $p$ 118,1990

5. Gustilo, R.B. and Anderson, J.T. Prevention of infection in the treatment of one thousand and twenty-five open fractures of long bones; retrospective and prospective analysis. J Bone Joint Surg, 58A:453-458, 1976.

6. Neer, C.S.; Grantham, S.A. and Shelton M.L. Supracondylar fracture of the adult femur. JBJS, Vol 49(A):591-613, 1967.

7. Sanders, R., Swiontkowski, M., Rosen, H. and Helfet, D. Double plating of communited, unstable fractures of the distal part of the femur. $J$ Bone Joint Surg, 73A:341-346, 1991.

8. Kregor, P.J.; Hughes,J.L. and Cole, P.A. Fixation of distal femoral fractures above total knee arthroplasty utilizing the Less Invasive Stabilization System (LISS). Injury, 32: 64-75, 2001.

9. Shandelmaier, P.; Partenhemer, A.; Koanemann, B. et al. Distal femoral fractures and LISS - stabilization. Injury, 32SC55-63, 2001.

10.Schutz, M.; Muller, M.; Krettek, C.; Hontzsch, D.; Regazzoni, P. and Ganz, R. Minimally invasive fracture stabilization of distal femoral fractures with the LISS: a prospective multicenter study. Results of a clinical study with special emphasis on difficult cases. Injury, 32(Suppl. 3),SC48SC54, 2001. 